\title{
MATERIALES DIDÁCTICOS DIGITALES: UN RECURSO INNOVADOR EN LA DOCENCIA DEL SIGLO XXI
}

DIGITAL DIDACTIC MATERIALS: AN
INNOVATIVE RESOURCE IN THE TEACHING OF THE
21ST CENTURY

Carolina Real Torres

Profesora Titular de Filología Latina.

Departamento de Filología Clásica, Francesa, Árabe y Románica.

Universidad de La Laguna (Tenerife). España. E-mail: carrel@ull.edu.es ORCID: https://orcid.org/0000-0002-2236-4265

Recepción: 09/11/2018 Aceptación: 12/12/2018 Publicación: 28/06/2019

Citación sugerida:

Real Torres, G. (2019). Materiales Didácticos Digitales: un recurso innovador en la docencia del siglo XXI. 3C TIC. Cuadernos de desarrollo aplicados a las TIC, 8(2), 12-27. doi: http://dx.doi. org/10.17993/3ctic.2019.82.12-27 


\section{RESUMEN}

El imparable progreso tecnológico que sacude nuestra sociedad ha llegado a las aulas académicas y, con él, el desarrollo de nuevos métodos de enseñanza. El avance de las TIC ha modificado la forma de elaborar, adquirir y transmitir conocimientos, promoviendo el uso de herramientas innovadoras para el aprendizaje. Dentro del amplio abanico de las nuevas posibilidades educativas que nos brindan estas herramientas, los materiales didácticos digitales de acceso abierto adquieren una importancia vital como fuente de información y eje del desarrollo de nuevos métodos pedagógicos.

En este sentido, el propósito de este trabajo es presentar un modelo de integración de MDD (Material Didáctico Digital), destacando los usos didácticos posibles que ofrecen estos recursos, y promover su diseño como estrategia dinámica para el trabajo autónomo, motivación y mejora del rendimiento académico del alumnado.

\section{PALABRAS CLAVE}

Materiales didácticos digitales, TIC (Tecnologías de la Información y la Comunicación), REA (Recursos Educativos en Abierto), Herramientas de Autor, Innovación Educativa. 
Ed. 29 Vol. 8 N. ${ }^{\circ} 2$ Junio - Septiembre 2019

DOI: http://dx.doi.org/10.17993/3ctic.2019.82.12-27

\section{ABSTRACT}

The unstoppable technological progress that shakes our society has reached the academic classrooms and, with it, the development of new teaching methods. The advance of ICT has modified the way of elaborating, acquiring and transmitting knowledge, promoting the use of innovative tools for learning. Within the wide range of new educational possibilities offered by these tools, open access didactic materials in digital format acquire vital importance as a source of information and as the axis for the development of new pedagogical methods.

In this sense, the purpose of this paper is to present a model of integration of MDD (Digital Didactic Materials), highlighting the possible didactic uses offered by these resources, and promote its design as a dynamic strategy for selfemployment, motivation and performance improvement student's academic.

\section{KEYWORDS}

Digital Didactic Materials, ICT (Information and Communication Technologies), OER (Open Educative Resources), Authoring Tools, Educational Innovation. 


\section{INTRODUCCIÓN}

La creación de materiales educativos es un aspecto clave de la incidencia de las TIC y del mundo digital en general en el actual panorama educativo (Rodríguez, 2005). Su preparación para el proceso de enseñanza-aprendizaje constituye el eje central de cualquier estrategia pedagógica debido a las numerosas posibilidades didácticas que ofrecen para trabajar contenidos no sólo conceptuales, sino también procedimentales y actitudinales, contribuyendo a la adquisición de competencias.

Los recursos digitales surgen en las últimas décadas como un medio de expresión y creación a través de un nuevo lenguaje basado en la imagen, el sonido y la interactividad, tres elementos que refuerzan la comprensión, la creatividad y la motivación de los estudiantes (García-Valcárcel, 2016). Los medios audiovisuales suponen una forma de comunicación multisensorial generadora de una multiplicidad de códigos que inciden sobre diversos sentidos y formas de percepción, formando un lenguaje de síntesis adoptado por los jóvenes como nuevo medio de comunicación. Los estudiantes que hoy en día llenan nuestras aulas quieren aprender de manera diferente.

En respuesta a esta demanda, la inmediatez y la espontaneidad, así como la colaboración y reutilización de materiales, se han convertido en rasgos de las nuevas formas de enseñanza (García-Valcárcel, 2016). Nuestros estudiantes están acostumbrados a los estímulos audiovisuales y a la versatilidad de la comunicación de la información, sobre todo a la inmediatez a la hora de acceder a ella, por lo que encuentran los recursos educativos tradicionales poco motivadores (Ortiz, 2006: 37-38). Esta nueva generación, ávida de tecnología, exige no sólo un cambio en los contenidos y en la forma en la que éstos se presentan, sino, además, quieren soluciones instantáneas a sus necesidades y un acceso actualizado a la información, más allá de los horarios y espacios físicos de los Centros. En esta línea, varios estudios recientes confirman no sólo la orientación positiva de los actuales estudiantes hacia la tecnología, los medios audiovisuales y el uso de dispositivos móviles, sino también la absoluta mayoría en la demanda de actividades on-line en su desarrollo formativo (Dahlstrom, 2015).

La integración de las TIC en esta nueva sociedad, infoxicada por el exceso de información, determina el éxito o fracaso del alumnado por su capacidad de seleccionar las fuentes más relevantes, por lo que las Universidades tendrán que preparar futuros docentes dedicados a la producción y desarrollo de sistemas basados en las TIC, que servirán para generar nuevo conocimiento, pues, como afirma 
Cantón (2000: 451), "Lo que añadirá valor a una persona es su capacidad para introducir una mejora en el producto o en el servicio, su capacidad de aprender de las innovaciones de otros, y su capacidad de adaptación a situaciones imprevisibles". Ante este panorama, se hace necesario la búsqueda de nuevos escenarios de aprendizaje y de nuevos mecanismos de distribución de contenidos. Contemplar las competencias digitales de los estudiantes como vector de impacto en el diseño de la experiencia digital es un elemento clave para evitar una brecha que puede no ser percibida inicialmente (Gisbert y Esteve, 2011: 49).

Este nuevo enfoque educativo exige, asimismo, un cambio de rol del docente, que se convierte en un curador o gestor de contenidos, creando, pero, ante todo, seleccionando y organizando todo tipo de material en red (Mena, 2014: 3). Dado que la integración curricular de las TIC es una cuestión pedagógica y no sólo técnica, la disponibilidad de materiales y la formación del profesorado son condiciones decisivas para hacer posible su integración. Sin embargo, la elaboración de materiales educativos es un proceso complejo que implica, por un lado, el diseño de materiales informáticos y, por otro, la elaboración de materiales educativos. Como observan O'Farrill y Tunis (2008: 61), "por una parte tendremos que utilizar y desarrollar el conjunto de técnicas y recursos propios de la informática (recursos de navegación, enlaces internos y externos, iconos, scripts, multimedias, etc.); y por la otra incorporamos los elementos o dimensiones propias de todo material destinado a la enseñanza y el aprendizaje: objetivos, actividades, contenidos, etcétera". Estos materiales didácticos son gestionados por el profesorado en función de sus competencias, para lo cual la formación y actualización del mismo es una cuestión de enorme relevancia (Livingstone, 2012: 18).

Dado que la integración curricular de las TIC es una cuestión pedagógica y no sólo técnica, la disponibilidad de materiales y la formación del profesorado son condiciones decisivas para hacer posible su integración.

El diseño de los materiales didácticos con fines educativos exige que se elaboren o se seleccionen para ser usados en una actividad educativa mediada por las TIC, debiendo cumplir con los criterios derivados de la didáctica, tales como la interactividad, la motivación, la creatividad, la colaboración y la representación del conocimiento (García-Valcárcel, 2016). En este sentido, las herramientas de autor pueden considerarse un nuevo tipo de software cuyo objetivo es facilitar la creación, 
publicación y gestión de los materiales educativos en formato digital. Estas herramientas han generado productos educativos muy importantes, tanto por su diseño como por sus posibilidades didácticas, reduciendo, además, el esfuerzo necesario del docente para producir software, guiándole y ofreciéndole elementos predefinidos (Murray, et al., 2003: 341 s.).

La principal diferencia respecto a los materiales educativos más tradicionales radica en la utilización de herramientas digitales que favorecen el uso autónomo por parte de los estudiantes y la interacción, una característica fundamental de los nuevos medios; asimismo, suelen conllevar un componente de gamificación, por lo que resultan altamente motivadores; y, por último, actúan también como herramientas de evaluación.

Su implementación en el curriculum plantea romper las barreras espaciotemporales que dominan la enseñanza tradicional ofreciendo materiales de acceso abierto en un entorno más flexible (Sevillano, 2015). En este sentido, los materiales digitales didácticos (MDD) son siempre reutilizables, pero, además, deben ser de carácter abierto para que otros docentes puedan adaptarlos a sus necesidades. A la hora de utilizarlos en la práctica diaria advertimos que ofrecen una mayor flexibilidad que los materiales tradicionales impresos en la medida en que son más susceptibles de ser adaptados y reutilizables para así adecuarlos a las necesidades concretas de un aula en particular o de nuestros intereses como docentes.

La contextualización es otro elemento importante a tener en cuenta. Los MDD no deben contener únicamente recursos digitales, sino que hay que considerar las formas en que son contextualizados para un uso educativo concreto, contemplando su función y adaptabilidad en unidades mayores, pues, como señala M. Area Moreira, "todo material didáctico digital debe estar al servicio del planteamiento pedagógico del curso o programa en el que se usará y debe ser utilizado como un medio o recurso para el logro de objetivos educativos" (Area, 2003: 36).

Por último, la creación de MDD conlleva una serie de ventajas, entre las que destacamos el fomento del aprendizaje activo, pues se logra que el discente se implique al reconocer los beneficios personales y la utilidad de su aprendizaje, convirtiéndose en responsable de la construcción de su conocimiento y de la elección de los medios más idóneos para ello. En este sentido, el empleo de metodologías activas permite la adquisición de las competencias digitales a través del trabajo en grupo mejorando 
el proceso de aprendizaje y el rendimiento del alumnado, pues, como apuntan Rodríguez y Espinoza (2017), "un trabajo hecho en un grupo de forma colaborativa tiene un resultado más enriquecedor que el que tendría la suma del trabajo individual".

\section{DESARROLLO}

\subsection{OBJETIVOS}

El objetivo principal de nuestra propuesta es la creación de Materiales Didácticos Digitales (MDD) a partir de aplicaciones informáticas de fácil acceso y diseño, destacando la importancia de las herramientas de autor para el desarrollo del proceso de enseñanza y aprendizaje. Se pretende facilitar el aprendizaje significativo y autónomo, así como la adquisición de competencias digitales del alumnado de la asignatura "Innovación docente e investigación educativa en la enseñanza de la lengua y la literatura (castellana, latín y griego)" del Máster en Formación del Profesorado de Educación Secundaria Obligatoria y Bachillerato, Formación Profesional y Enseñanzas de Idiomas (Interuniversitario) por la Universidad de La Laguna, con el fin último de poder llevar a cabo la creación y elaboración de sus propios MDD relacionados con el ámbito de las Humanidades.

Entre los objetivos específicos se contemplan:

- Capacitar en la planificación, diseño y elaboración de MDD.

- Conocer y evaluar la viabilidad de las principales herramientas y aplicaciones (Apps) disponibles para la elaboración de los MDD.

- Capacitar en el uso de técnicas de innovación para transformar las guías docentes con la inclusión de MDD.

- Capacitar en el control del uso de los Materiales Didácticos Digitales en el aula. 


\subsection{MÉTODO}

Para el desarrollo del proyecto se promueve, por tanto, la creación de materiales didácticos propios, conocidos como herramientas de autor, y la presentación de las principales herramientas gratuitas para la creación de contenidos con la finalidad de valorar su eficacia y viabilidad pedagógica. Para este nuevo tipo de software se sugieren distintos programas gratuitos o aplicaciones web que el alumnado deberá elegir conforme a sus necesidades y su nivel de competencias y conocimientos. En este punto el papel del tutor como facilitador cobra vital importancia, ya que permite orientar al alumnado entre las múltiples posibilidades disponibles en red, capacitándolo para optimizar los recursos y fomentando su pensamiento crítico acerca de la funcionalidad de las herramientas empleadas.

Para alcanzar los objetivos propuestos se ha buscado fomentar el aprendizaje autodirigido en el que los estudiantes trabajan en equipo en entornos virtuales. La metodología de aprendizaje basada en proyectos supone, en este caso, promover o incentivar la selección de aplicaciones o herramientas según las necesidades de cada docente o, como es el caso, del futuro docente para la elaboración de su MDD. De esta forma se genera una autonomía que le ayudará a resolver cualquier situación independientemente de los recursos materiales o virtuales de los que disponga en la red.

La metodología de aprendizaje basada en proyectos supone promover o incentivar la selección de aplicaciones o herramientas según las necesidades de cada docente o del futuro docente para la elaboración de su MDD.

Se propone, a continuación, la creación de distintos materiales destinados a formar una colección o secuencia de actividades alojadas en una plataforma digital que permita crear, gestionar y almacenar diferentes tipos de materiales, además de compartir contenidos para su posterior visualización. Este tipo de plataformas resultan idóneas para el trabajo colaborativo y el intercambio de información. Por otro lado, dado que las colecciones formadas por distintas actividades interactivas y sus posibles combinaciones podrían considerarse como piezas de un rompecabezas destinadas a encajar perfectamente unas con otras, la elección de las actividades debe responder a un criterio didáctico o "pertinencia educativa" (Rodríguez, 2005); es decir, eligiendo recursos que puedan cumplir una función similar y combinándolos de manera coherente para alcanzar los objetivos previstos. 
Finalmente, los resultados son compartidos en la red a través de comunidades educativas, lo que permite obtener una valoración crítica del trabajo llevado a cabo por parte del resto de grupos. Las redes de comunicación, y la apuesta por la colaboración a través de las mismas, posibilitan el nacimiento de nuevos proyectos para compartir los recursos audiovisuales, añadiendo interactividad y permitiendo su exposición en Internet.

\subsection{PROYECTO}

La experiencia -como hemos señalado- se ha llevado a cabo en la asignatura "Innovación docente e investigación educativa en la enseñanza de la lengua y la literatura (castellana, latín y griego)", una materia cuatrimestral correspondiente al Máster en Formación del Profesorado de Educación Secundaria Obligatoria y Bachillerato, Formación Profesional y Enseñanzas de Idiomas (Interuniversitario) por la Universidad de La Laguna, durante el curso académico 2017-2018. Para su desarrollo contamos con un total de 24 estudiantes, de los cuales, según una encuesta inicial, todos tienen un elevado gusto por la informática y un 80\% afirma tener conocimientos informáticos. Ante la pregunta "Elige entre las competencias de la asignatura la que consideres más importante para el futuro desarrollo de tu práctica docente", un 60\% seleccionó "Conocer y aplicar metodologías y técnicas básicas de investigación y evaluación educativas y ser capaz de diseñar y desarrollar proyectos de investigación, innovación y evaluación", y el 100\% afirmó estar dispuesto a introducir las TIC en sus clases.

Con este panorama tan favorecedor para los objetivos del proyecto, se procedió a la elección de la plataforma más adecuada para su desarrollo, resultando elegida Educaplay (https:// es.educaplay.com/), la cual permitió crear, alojar y publicar distintos materiales multimedia, clasificados por categorías según la edad y el nivel de estudios de los posibles usuarios e identificados por etiquetas según la temática. Estos materiales son de acceso abierto y gratuito, y no sólo pueden ser utilizados desde el ordenador o cualquier dispositivo móvil, sino también exportados a blogs o páginas web.

Los entornos colaborativos como Educaplay constituyen herramientas de almacenamiento, similares a cualquier otro repositorio de acceso abierto, y son, a la vez, herramientas de comunicación, pues constituyen un potente medio de difusión de materiales. Para cubrir las necesidades de una 
comunicación a tiempo real se empleó el chat del aula virtual de la asignatura y la comunidad de Google+ creada para la ocasión.

La dinámica empleada en el aula consistió en dividir al alumnado en grupos de cuatro para la creación de MDD y la posterior formación de colecciones de actividades de una forma organizada, pedagógica y visualmente atractiva. Se atendió principalmente a la organización de las mismas por temas y en niveles significativos desde un punto de vista educativo, distinguiendo un orden secuencial cuando respondían a una dificultad progresiva o libre cuando se quería fomentar el aprendizaje autónomo, en el que el alumnado puede elegir los contenidos y recursos que más le interesan y en el orden que considera más conveniente.

Las herramientas empleadas para la creación de materiales fueron videoquiz, ruletas de palabras, cuestionarios, crucigramas, mapas interactivos, sopas de letras y presentaciones. La elección de estas herramientas fue opcional por parte del alumnado, excepto el videoquiz, que, por su potencialidad didáctica, se aconsejó que todos los grupos lo incluyeran. Esta excepción se basa en las ventajas que ofrece como vídeo interactivo, al permitir la asociación de contenidos de diversa naturaleza a lo largo de su línea narrativa (García-Valcárcel, 2008; García Matamoros, 2014). Los videoquiz o hipervídeos se caracterizan tanto por ampliar la información de la secuencia del video conductor, como por su adaptabilidad al ritmo de cada estudiante y a su eficacia motivadora, pues no sólo transmiten información exhaustiva sobre un tema, sino que abren interrogantes, suscitan problemas, generan dinámicas participativas y aumentan la atención del alumnado (Prats, 2003: 62 y 67; Romero, 1996: 130-132).

Por último, en la valoración de la actividad se contemplan formularios de autoevaluación, que permiten al alumnado tener conocimiento de su progreso, una encuesta de satisfacción sobre los resultados obtenidos y una evaluación final donde se valora el nivel de contenidos y la relevancia de la información, el nivel de creatividad y la coherencia en la secuencia de actividades. La calidad de las herramientas de autor se juzga tanto desde el punto de vista técnico como pedagógico, atendiendo a su diseño, compatibilidad, reutilización y adecuación al desarrollo del curriculum escolar. La suma de estos datos nos ofrecerá una valoración objetiva sobre su potencial didáctico y, en consecuencia, sobre su integración curricular. 


\subsection{RESULTADOS}

En cuanto a los resultados de esta experiencia, hemos podido comprobar que el nivel de habilidades y conocimientos que el autor de los materiales didácticos requiere es mínimo. La complejidad del proceso de producción puede ser reducida mediante herramientas apropiadas, de fácil manejo, preferentemente online (no requieren la instalación de ningún programa en nuestro ordenador), gratuitas, y con la ayuda del profesorado, que, en este caso, actúa como guía de todo el proceso de formación. Hay herramientas cada vez más fáciles de utilizar por el usuario, pero del docente depende capacitar al alumnado para seleccionar las aplicaciones idóneas para crear su MDD.

La flexibilidad de estos materiales se extiende a su uso por parte de los propios estudiantes, ya que pueden reconfigurarlos, modificando o ampliando, en otro contexto, cumpliendo de esta manera con una de las características principales de los MDD, su reutilización. En este sentido, la mayoría reconoció la utilidad de estas herramientas y mostraron al final del proceso haber adquirido la capacidad suficiente para crear sus propios materiales, una parte fundamental en la gestión y organización de sus clases al incorporarse al mercado laboral.

Por otra parte, mediante el trabajo colaborativo los estudiantes desarrollaron su capacidad de aprender a aprender y de resolver problemas trabajando en grupo, tomando conciencia de que el aprendizaje es un proceso que dura toda la vida. A la naturaleza esencialmente social de este tipo de aprendizaje se suma la experiencia de realizar vídeos didácticos para su uso en el aula con unos objetivos pedagógicos bien definidos, un proceso de aprendizaje creativo que permite poner en práctica las ideas del alumnado sobre cómo enseñar determinados contenidos.

Podemos afirmar que la eficacia de una experiencia de innovación educativa está relacionada con la forma en la que se lleve a cabo su aplicación en el contexto del aula y con la presencia de elementos significativos que indiquen una relación directa entre sus contenidos, el programa de la asignatura y quiénes lo imparten. A este respeto, los resultados demuestran que el papel del profesorado en los ambientes virtuales es muy importante para generar una nueva cultura en el aprendizaje y la praxis docente. 
Finalmente, se observan indicios de mejora en los resultados de aprendizaje y en la motivación de los estudiantes debido, sin duda, a un tipo de enseñanza más interactiva y un aprendizaje más significativo, pues las TIC ayudaron a diseñar actividades didácticas que contribuyeron a implicar a los estudiantes, quienes mostraron una gran predisposición a participar en actividades colaborativas, generando un ambiente de comunicación entre sus compañeros, y a difundir sus resultados en las redes sociales. Hemos comprobado una vez más cómo la interacción social favorece el aprendizaje.

\section{CONCLUSIONES}

La implementación del desarrollo de MDD en el proceso de enseñanza-aprendizaje busca, ante todo, que el futuro docente sea capaz de generar sus propios materiales basados en modelos pedagógicos específicos y sea capaz de enfrentarse a nuevas situaciones y nuevos escenarios de aprendizaje para resolver con éxito su futura práctica docente.

El éxito del aprendizaje basado en proyectos y el trabajo colaborativo llevado a cabo en el aula ha propiciado un ambiente crítico y enriquecedor y ha proporcionado a los estudiantes las herramientas necesarias para poder innovar en cualquier asignatura que vayan a impartir en el futuro, una vez rota la brecha tecnológica que han superado. Los resultados derivados de la encuesta de satisfacción revelan que se ha alcanzado un alto grado de éxito respecto a los objetivos iniciales.

Por consiguiente, la creación de MDD facilita desarrollar en los alumnos y alumnas diversas destrezas relacionadas con un aprendizaje autónomo y significativo, trabajar de forma colaborativa, utilizar diversos recursos tecnológicos y compartir los resultados obtenidos en las redes, un medio muy cercano a su realidad fuera de las aulas.

La creación de MDD facilita desarrollar en los alumnos y alumnas diversas destrezas relacionadas con un aprendizaje autónomo y significativo.

Las conclusiones a las que llegamos sobre las herramientas empleadas son, por un lado, que el vídeo digital amplía las funciones tradicionales del vídeo en la enseñanza permitiendo una mayor 
interactividad y, como resultado, está revitalizando el uso de la comunicación audiovisual desde una perspectiva educativa; por otro, las comunidades virtuales y plataformas educativas de acceso abierto posibilitan la publicación de materiales para ser compartidos, a la vez que fomentan nuevas vías de comunicación dentro de la cultura de las redes sociales. En este contexto, los MDD impulsados por las redes sociales permiten reconstruir y generar nuevos contenidos a través de herramientas colaborativas.

La gran complejidad de formar en la competencia digital a nuestro alumnado es la de buscar respuestas para un mundo que todavía desconocemos. Tenía razón Zygmunt Bauman (2015) cuando afirmó que nuestra sociedad era una suerte de modernidad líquida, en tanto que los valores y aspiraciones de la humanidad se transforman de manera vertiginosa. Por tanto, las Universidades tendrán que preparar profesionales capaces de elaborar y diseñar nuevos materiales didácticos que se ajusten a sus necesidades y a los nuevos tiempos. Surge, por tanto, la necesidad de la inclusión de las competencias digitales en el desarrollo de las asignaturas del Máster en Formación del Profesorado de Educación Secundaria Obligatoria y Bachillerato, Formación Profesional y Enseñanzas de Idiomas (Interuniversitario) por la Universidad de La Laguna, y su aplicación en las prácticas docentes curriculares realizadas en los Centros de Secundaria y Bachillerato.

El alumnado de Máster, con una edad comprendida entre 24 y 30 años, pertenecen a la generación de los millennials, usando la terminología acuñada por Howe y Strauss (2000). No han nacido con la tecnología debajo del brazo, pero sí manejan sin dificultad alguna cualquier dispositivo móvil o aparato informático, interactuando con ellos de forma casi intuitiva. Estos jóvenes, muy interesados en cualquier novedad tecnológica, sabrán transmitir, sin duda alguna, su conocimiento y su entusiasmo a las nuevas generaciones. 


\section{REFERENCIAS BIBLIOGRÁFICAS}

Area Moreira, M. (2003). De los webs educativos al material didáctico web. Revista Comunicación y Pedagogía, (188), 32-38.

Bauman, Z. (2015). Modernidad líquida. Fondo de cultura económica.

Cantón, I. (2000). Las tecnologías como utopía en la sociedad de la información y el conocimiento y su incidencia en las instituciones educativas. Las organizaciones educativas en la sociedad neoliberal. Granada: Grupo Editorial Universitario, 445-461.

Dahlstrom, E. (2015). Educational technology and faculty development in higher education. Educause Center for Analysis and Research. Recuperado de: http://net. educause. edu/ir/library/pdf/ ers 1507.pdf

García Matamoros, M.A.(2014). Uso instruccional del video didáctico. Revista de investigación,38(81), 43-68.

García-Valcárcel Muñoz-Repiso, A. (2008). El hipervídeo y su potencialidad pedagógica. Revista Latinoamericana de Tecnología Educativa-RELATEC, 7(2), 69-79.

García-Valcárcel Muñoz-Repiso, A. (2016). Recursos digitales para la mejora de la enseñanza y el aprendizaje. Recuperado de: https://gredos.usal.es/jspui/bitstream/10366/131421/1/ Recursos\%20digitales.pdf

Gisbert, M. y Esteve, F. (2011). Digital Learners: la competencia digital de los estudiantes universitarios. La Cuestión Universitaria 6(7), 48-59.

Howe, N. y Strauss, W. (2000). Millennials Rising: The Next Great Generation. Vintage.

i Prats, J. F. (2003). Educación en medios y competencia emocional. Revista Iberoamericana de Educación, 32(1), 49-69.

Livingstone, S. (2012). Critical reflections on the benefits of ICT in education. Oxford Review of Education, 38(1), 9-24. 
Mena Muñoz, S. (2014). Herramientas contra la infoxicación en los Social Media: los "Content Curators". Ámbitos. Revista internacional de comunicación, (24), 1-12.

Murray, T., Blessing, S., y Ainsworth, S. (Eds.). (2003). Herramientas de creación para entornos de aprendizaje de tecnología avanzada: hacia un software educativo adaptativo, interactivo e inteligente rentable. Springer Science y Business Media.

O’Farrill, J. L. M. y Tunis, E. H. (2008). Las herramientas de autor en el proceso de producción de cursos en formato digital. Pixel-Bit. Revista de medios y educación, (33), 59-72.

Ortiz Colón, A. (2006). La implantación de las TIC o el uso de internet en la escuela. Innovación educativa, (16), 31-45.

Rodríguez Illera, J. L., Escofet, A., y Azzato, M. (2005). Un sistema abierto para la creación de contenidos educativos digitales. RED. Revista de Educación a Distancia, (IV). Recuperado de: http://www.um.es/ead/red/M4/

Rodríguez Zamora, R., y Espinoza Núñez, L. A. (2017). Trabajo colaborativo y estrategias de aprendizaje en entornos virtuales en jóvenes universitarios. RIDE. Revista Iberoamericana para la Investigación y el Desarrollo Educativo, 7(14), 86-109.

Romero Tena, R. (1996). Utilización didáctica del vídeo. II Fornadas sobre Medios de Comunicación, Recursos y Materiales para la Mejora Educativa. (127-149). Sevilla: Ayuntamiento de Sevilla, Centro Municipal de Investigación y Dinamización Educativa: Universidad de Sevilla, Secretariado de Recursos Audiovisuales y Nuevas Tecnologías, 127-149.

Sevillano-García, M. L. (2015). El contexto socioeducativo de la ubicuidad y la movilidad. En E. Vázquez-Cano y M. L. Sevillano García (Eds.), Dispositivos digitales móviles en educación. El aprendizaje ubicuo. Madrid: Narcea. 